\title{
Odontoma em paciente odontopediátrico: diagnóstico e tratamento. Relato de caso clínico
}

\author{
Odontoma in pediatric patient: diagnosis and treatment. A case report
}

\begin{abstract}
Marcelle Danelon
Aluna de Doutorado do Programa de Pós-Graduação em Ciência Odontológica Área de Concentração: Saúde Bucal da Criança da Faculdade de Odontologia da Universidade Estadual Paulista "Júlio de Mesquita Filho" - FOA/UNESP

Michele Mauricio Manarelli Aluna de Doutorado do Programa de Pós-Graduação em Ciência Odontológica Área de Concentração: Saúde Bucal da Criança da Faculdade de Odontologia da Universidade Estadual Paulista "Júlio de Mesquita Filho" - FOA/UNESP

Luciene Pereira de Castro Aluna de Doutorado do Programa de Pós-Graduação em Ciência Odontológica Área de Concentração: Saúde Bucal da Criança da Faculdade de Odontologia da Universidade Estadual Paulista "Júlio de Mesquita Filho" - FOA/UNESP
\end{abstract}

RoBson FrEDERICO CUNHA Professor Adjunto da Faculdade de Odontologia da Universidade Estadual Paulista "Júlio de Mesquita Filho" - FOA/UNESP

CÉlio Percinoto Professor Titular da Faculdade de Odontologia da Universidade Estadual Paulista "Júlio de Mesquita Filho" - FOA/UNESP

\begin{abstract}
RESUMO
Odontoma é um termo que se refere a um tumor benigno de origem odontogênica e de natureza mista, constituído por componentes epiteliais e mesenquimais. Histologicamente é composto de várias formas dentárias, entre as quais se encontra esmalte, dentina, cemento e, em alguns casos, tecido pulpar. Clinicamente, apresenta crescimento lento e é assintomático, sendo geralmente descoberto por meio de exames radiográficos de rotina. Paciente do gênero masculino, três anos de idade, compareceu à Bebê Clínica da Faculdade de Odontologia de Araçatuba da Unesp queixando-se de "bolinha perto dos dentes". Durante a anamnese, a mãe relatou que a lesão foi observada logo após um trauma, e que evoluiu em menos de um mês. No exame clínico intraoral observou-se que havia uma lesão ulcerada com $0,8 \mathrm{~cm}$ de diâmetro, localizada na região inferior e anterior entre os dentes 81 e 82, notando-se também a distalização das coroas. Após o exame radiográfico, notou-se uma área radiolúcida e afastamento das raízes. Na ausência de características radiográficas e clínicas que conduzissem a um caso de odontoma, o diagnóstico diferencial foi de lesão periférica de células gigantes e granuloma piogênico. Assim, realizou-se punção da área. Entretanto, não havendo presença de líquido, foi realizada a remoção cirúrgica da lesão, seguida pelo estudo histopatológico, o qual apresentou como diagnóstico definitivo um caso sugestivo de odontoma em formação.
\end{abstract}

Palavras-chave: ODONTOMA, ODONTOPEDIATRIA, DIAGNÓSTICO, TRATAMENTO. 
Marcelle Danelon et al.

\begin{abstract}
Odontoma is a term that refers to a benign tumor of odontogenic and mixed nature, composed of epithelial and mesenchymal components. Histologically, they are compounds of different configurations including dental enamel, dentin, cementum and in some cases the pulp tissue. A slow growing asymptomatic tumor, odontoma is usually discovered through routine radiographic examination. A 3-year old male patient sought care at the School of Dentistry's Baby Clinic (UNESP-Araçatuba), complaining of "small ball close to the teeth." During the interview, the mother reported that the lesion was observed soon after a trauma, and evolved in less than one month. An ulcerated lesion with a $0.8 \mathrm{~cm}$ diameter was found during intraoral clinical examination. It was located in the inferior and anterior region of the mouth, between teeth 81 and 82 , and there was also crown distalization. A radiographic examination showed a radiolucent area and root distance. In the absence of clinical and radiographic characteristics suggesting a case of odontoma, the differential diagnosis was peripheral giant cell lesion and pyogenic granuloma. So the area was punctured. Nonetheless, due to the absence of liquid, the surgical removal of the lesion was performed, followed by histological examination, which showed the definite diagnosis of a suggestive case of emerging odontoma.

Keywords: ODONTOMA, DENTISTRY, DIAGNOSIS, TREATMENT.
\end{abstract}

\section{INTRODUÇÃO}

Odontoma é classificado como o tipo mais comum de tumores odontogênicos, ${ }^{1,2}$ representando até $70 \%$ de todos os tumores odontogênicos encontrados. ${ }^{2,3,4}$ Apesar de sua classificação como tumores odontogênicos, alguns autores os consideram como malformações de desenvolvimento (hamartomas), em que todos os tecidos dentais estão representados e, como tais distúrbios de formação, passíveis de ocorrer sob uma série de fatores. ${ }^{5,6}$ São assintomáticos, podendo ser identificados pela falta de erupção, desvio da posição normal dos dentes ou em exames.

Várias hipóteses têm sido levantadas para explicar a etiologia desta patologia. Alguns autores sugerem que traumatismos e a presença de infecção local poderiam desencadear a formação de um odontoma. É cogitada, ainda, uma possível associação com fatores genéticos.$^{7,8}$ Segundo a Organização Mundial da Saúde (OMS), os odontomas são classificados em dois tipos distintos: odontoma composto e complexo. ${ }^{1}$

Radiograficamente o odontoma composto é uma malformação que surge de uma proliferação exorbitante da lâmina dentária na qual os tecidos dentais estão representados em um padrão ordenado, envoltos por uma zona radiolúcida formando estruturas semelhantes a pequenos dentes o que, muitas vezes, faz com que o diagnóstico dessa lesão seja puramente clínico e radiográfico. O odontoma complexo, por sua vez, surge de uma invaginação do epitélio no germe em desenvolvimento em um padrão onde os tecidos dentais representados estão desordenados, ou apresentam-se como um conglomerado de massa radiopaca bem delimitada envoltos por um halo radiolúcido, entretanto, sem que seja possível perceber semelhança com dentículos. . $^{9,10,11}$

Geralmente os odontomas estão localizados pericoronalmente a dentes impactados, mas também podem ser encontrados entre as raízes dentárias. Não mostram predileção por gênero e apresentam ocorrência levemente maior na região anterior da maxila. Verifica-se também uma tendência dos odontomas compostos ocorrer mais na maxila, e os complexos mais na mandíbula, assim como uma ocorrência maior de odontomas compostos. ${ }^{12}$

A análise histopatológica revela que os mesmos são muito semelhantes à estrutura dentária normal com a presença de esmalte, dentina, cemento e polpa. ${ }^{13,14}$ A principal diferença entre os dois tipos de odontoma é que o composto exibe um padrão de morfodiferenciação que resulta em uma lesão constituídapormúltiplosdentículos. Oodontoma complexo, por sua vez, é constituído por uma 
massa conglomerada de tecido dentário duro. Presença de espaços ou fendas contendo tecido pulpar, matriz de esmalte ou esmalte imaturo podem também ser encontrados. ${ }^{8} \mathrm{O}$ tratamento indicado para odontomas consiste na remoção cirúrgica, facilmente executada, uma vez que são separados do osso adjacente por cápsula fibrosa de tecido conjuntivo. Dessa forma não é esperada a recidiva da lesão, o que nos oferece um excelente prognóstico do tratamento. ${ }^{11} \dot{E}$ fundamental salientar que o odontoma pode assemelhar-se, principalmente radiograficamente a outras lesões neoplásicas ou císticas, assim é fundamental que a peça cirúrgica seja encaminhada para a análise histopatológica o que permitirá um diagnóstico definitivo e garantir um prognóstico ao paciente.

Assim, este artigo teve por objetivo relatar um caso de odontoma em formação em paciente pediátrico, assim como a análise radiográfica e histopatológica para se determinar a conduta ideal prestada ao caso.

\section{Relato de Caso}

Paciente do gênero masculino, 3 anos de idade, raça parda, procurou a Bebê Clínica da Faculdade de Odontologia de AraçatubaUNESP, encaminhado por uma cirurgiãdentista da própria cidade, para avaliação de uma lesão que a mãe do paciente descrevia como uma "bolinha perto dos dentes".

Durante a anamnese, a mãe relatou que, a lesão foi observada logo após um trauma e que evoluiu aproximadamente em menos de 1 mês. Durante o exame físico, o paciente não mostrava nenhuma alteração ganglionar e não apresentava sensibilidade dolorosa expontânea e provocada na área referida. Ao exame clínico intra-oral observou-se presença de lesão ulcerada com 0,8 centimetros de diâmetro, não pediculada, localizada na parte lingual da região inferior entre os dentes 81 e 82, notando-se também a distalização das coroas (Figura 1).

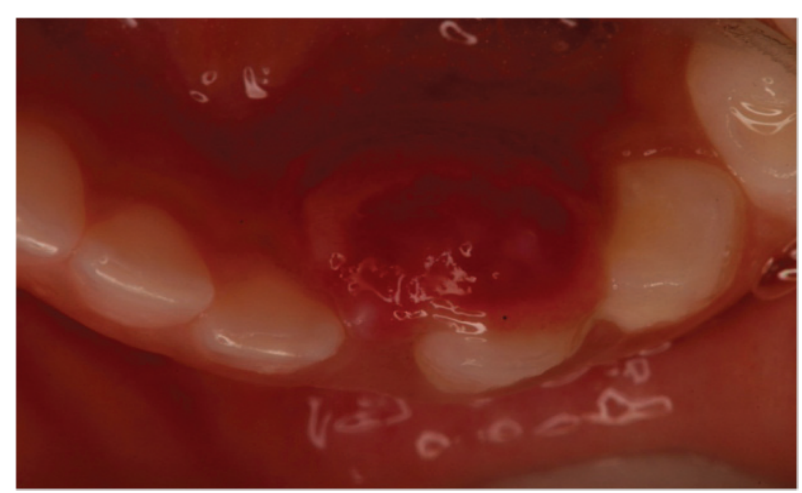

Figura1 - Aspecto da lesão e distalização das coroas

Ao exame radiográfico verificou-se uma área radiolúcida circunscrita entre os dentes 81 e 82 e afastamento das raízes dos mesmos. A hipótese diagnóstica foi lesão periférica de células gigantes e granuloma piogênico (Figura 2).

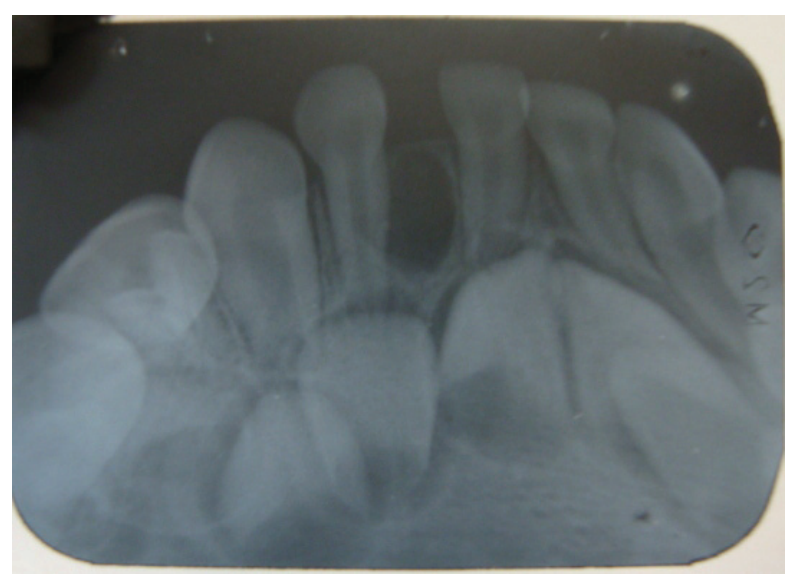

Figura 2 - Área radiolúcida circunscrita e afastamento das raízes

O diagnóstico definitivo foi determinado pelo exame histopatológico, através de biópsia excisional. Realizou-se punção da área, entretanto, não havendo presença de líquido, optou-se pela remoção cirúrgica da lesão (Figura 3). 


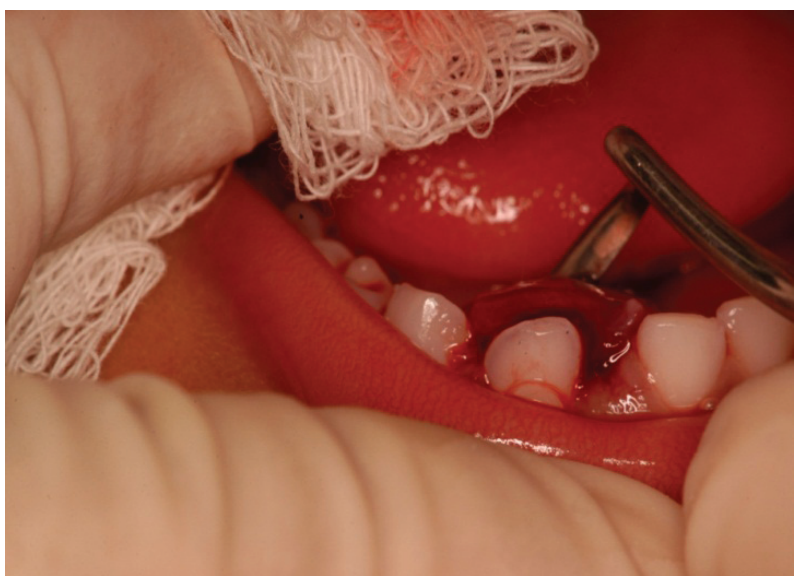

Figura 3 - Remoção cirúrgica da lesão

Após a remoção da lesão, a peça cirúrgica foi encaminhada para análise histopatológica, a qual apresentou como diagnóstico definitivo caso sugestivo de odontoma em formação (Figuras 4 e 5).

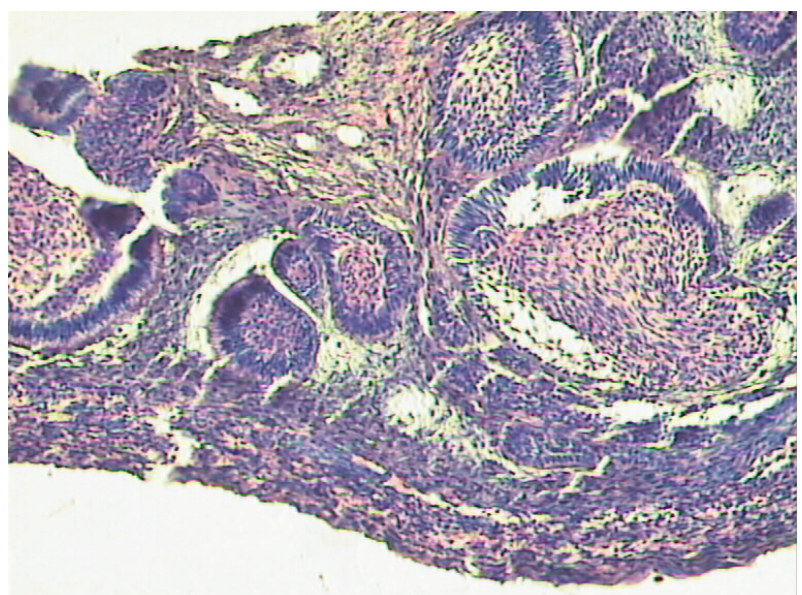

Figura 4 - Na microscopia foi possível observar áreas com formações de estruturas semelhantes à polpa dentária (1) com organização de células periféricas polarizadas (seta). Aumento original de 20x, coloração de H.E.

Os retornos foram seguidos de 7, 15, 30 e 60 dias, para novas avaliações. Observouse clinicamente cicatrização por segunda intenção. A mãe do paciente foi orientada a fazer o acompanhamento radiográfico durantes 6 meses para proservação cirúrgica (Figuras 6,7 e 8).

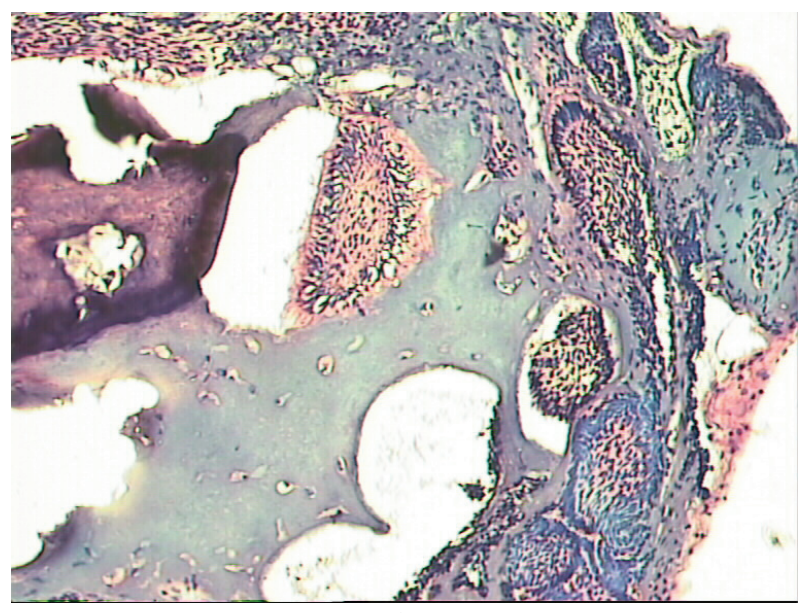

Figura 5: Em outros fragmentos da amostra enviada para análise microscópica observamos espaços formados por estruturas semelhantes a polpa (1), formação de matriz semelhante a dentina e/ou osteodentina (2) com áreas de mineralização (3). Aumento original de 20x, coloração de H.E.

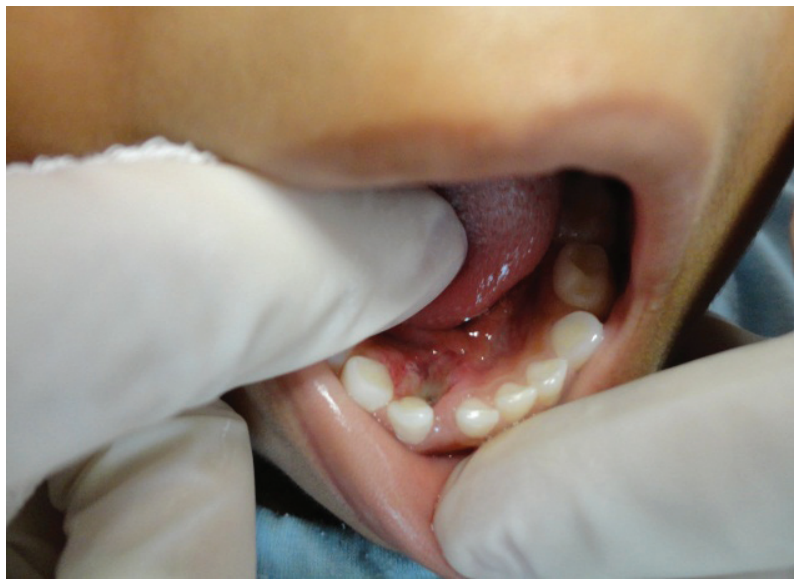

Figura 6 - Pós-operatório de 7 dias

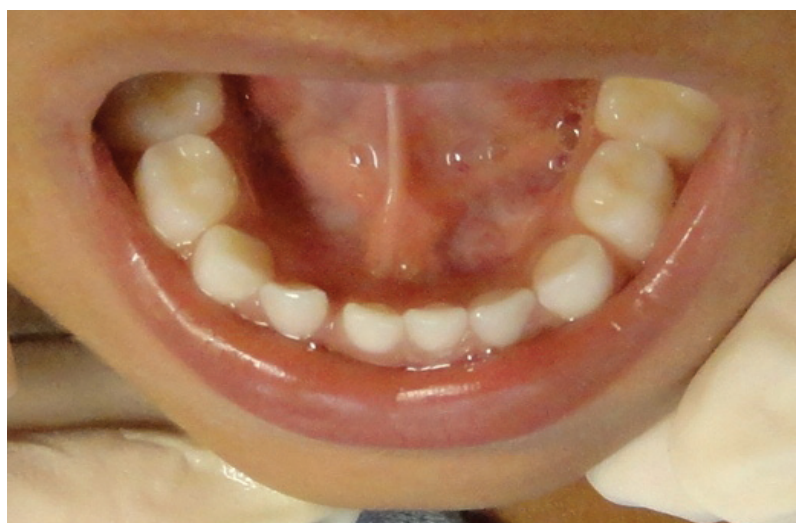

Figura 7 - Pós-operatório de 30 dias. 


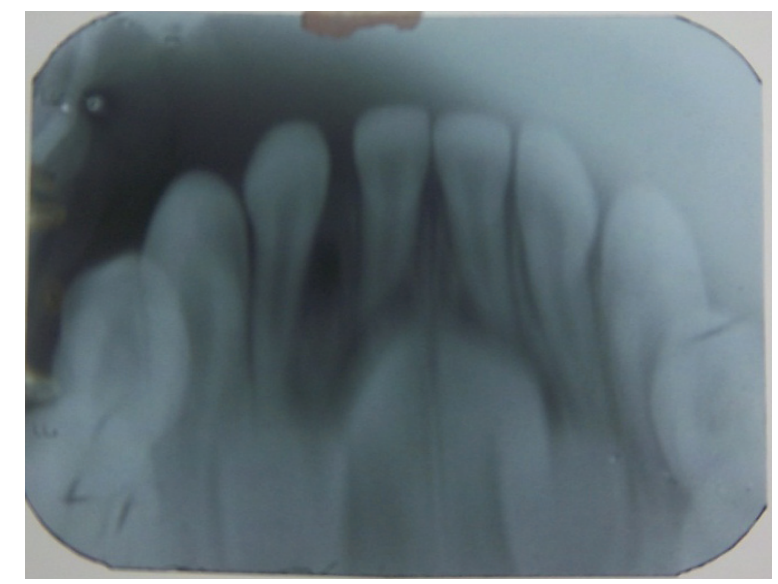

Figura 8 - Radiografia após 30 dias.

\section{Dıscussão}

Odontoma caracteriza-se por uma lesão de tumor odontogênico, embora seja considerada por diversos autores uma malformação hamartomatosa do que como uma neoplasia verdadeira. Apesar de a OMS classificar essa entidade como um tumor, os casos relatados se assemelham a alterações de desenvolvimento diagnosticadas após exames de rotina realizados pela não erupção dos dentes permanentes. ${ }^{5,6}$ Os odontomas são patologias assintomáticas, de evolução lenta, embora neste relato de caso, observou-se uma neoformação de rápido desenvolvimento, atingindo, na maioria dos casos, pequenas proporções, podendo passar despercebido e trazer prejuízo ao paciente tanto nas estruturas ósseas e dentárias, como provocando alterações na oclusão.

Segundo Cardoso et al. ${ }^{3}$ e Pires et al. ${ }^{15}$ algumas vezes, eles podem atingir grandes volumes, causando expansão das corticais ósseas e sensação dolorosa devido à compressão de estruturas nobres. Nos casos relatados, os odontomas foram de pequenas proporções e totalmente assintomáticos, tendo sido diagnosticados apenas em exames radiográficos de rotina a partir da investigação da não erupção dos elementos permanentes e tratamentos ortodônticos.
$\mathrm{Na}$ maioria dos casos, os odontomas são encontrados em pacientes jovens. Como relatado no presente caso clínico, podendose concordar com Cardoso et al. ${ }^{3}$ que também observaram a presença de odontomas, causando uma série de transtornos oclusais, como interferência no processo de erupção do dente, deslocamento e má formação dos dentes vizinhos e, em alguns casos, erupção ectópica. Observa-se nas figuras 1 e 2, o deslocamento dos dentes 81 e 82 , provocado pelo odontoma em formação. Pires et al. ${ }^{15}$, relataram ainda que a presença de diastemas persistentes, divergências do longo eixo de dente e assimetria facial podem relacionam-se à presença dessa patologia.

Os odontomas relatados são os mais comuns na literatura, pois são do tipo composto, porém a sua localização na região anterior da mandíbula não corrobora a localização mais frequentemente encontrada nesse tipo de tumor. Segundo Neville et al. ${ }^{1} \mathrm{e}$ Lukes et al. ${ }^{16}$, os odontomas se localizam com maior frequência na região anterior da maxila. O tratamento é cirúrgico conservador, com certa facilidade de clivagem pela presença de uma cápsula fibrosa semelhante ao folículo dentário, principalmente nos casos de odontomas complexos. Fatores anatômicos, entretanto, podem limitar o acesso à lesão e os riscos devem ser informados ao paciente antes da cirurgia.

No presente caso clínico, na ausência de características radiográficas e clínicas que conduzissem a um caso de odontoma, o diagnóstico diferencial foi como lesão periférica de células gigantes e granuloma piogênico e o laudo histopatológico indicou tratar-se de um odontoma em formação. É importante destacar que as peças devem ser sempre encaminhadas para a confirmação histopatológica visto que outras lesões semelhantes, como o odontoma ameloblástico, implicam em diferentes estratégias de tratamento. 
A remoção cirúrgica realizada como forma de tratamento para o caso relatado está de acordo com as condutas terapêuticas sugeridas por Cardoso et al. ${ }^{3}$, Blinder et al. ${ }^{17}$; Serra-Serra et al. ${ }^{18}$ Além da simples remoção do tumor, buscou-se o acompanhamento do caso. Até o último exame radiográfico de proservação realizado, não se observou presença de complicações e/ou recidiva, comprovando o baixo índice de recidiva dessa lesão e o sucesso do correto plano de tratamento realizado.

\section{Considerações Finais e Conclusão}

O odontoma é considerado uma malformação benigna relativamente comum e de fácil diagnóstico clínico e por imagens; geralmente assintomático; com evolução lenta, podendo causar retenção de dentes permanentes adjacentes. Seu tratamento de escolha consiste na excisão cirúrgica, na qual deve ser realizada a completa remoção e curetagem tão logo sejam diagnosticados, a fim de promover a reabilitação precoce e/ou evitar futuras sequelas, tanto oclusais quanto da formação de outras lesões associadas.

O prognóstico neste caso clínico foi favorável, pois a lesão não apresentou características de malignização e a recidiva é muito rara. A proservação radiográfica é importante para assegurar o sucesso do tratamento.

\section{REFERÊNCIAS}

1. Neville BW, Damm DD, Allen CM, Bouquot JE. Patologia oral \& maxilofacial. 2 ed. Rio de Janeiro: Guanabara Koogan; 2008.

2. Freitas DA, Freitas VA, Mol VC, Mana Neto L, Mol VC. Elemento dental impactado por odontoma composto. Rev Bras Cir Cabeça Pescoço. 2009 jul-set; 38 (3): 198-9.

3. Cardoso LC, Miyahara GI, Magro Filho O, Garcia Júnior IR, Soubhia AMP. Odontoma combinado associado a dentes não irrompidos: relato de casos clínicos. Rev Odont Araçatuba. 2003 ago-dez; 24 (2): 47-51.
4. Silva LF, David, L, Ribeiro D, Felino A. Odontomas: a clinicopathologic study in a portuguese population. Quintessence Int. 2009 jan; 40 (1): 61-72.

5. Tommasi AF. Diagnóstico em patologia bucal. $2^{a}$ ed. São Paulo: Pancast; 1998.

6. Teruhisa U, Murakami J, Hisatomi M, Yanagi Y, Asaumi J. A case of unerupted lower primary second molar associated with compound odontoma. Open Dent J. 2009 aug; 3: 173-6.

7. Lopez-Areal L, Silvestre DF, Gil LJ. Compound odontoma erupting in the mouth: 4-year followup of a clinical case. J Oral Pathol Med. 1992 jul; 21 (6): 285-8.

8. Amorim RFB, Queiroz SBF, Medeiros AMC, Souza LB. Odontoma complexo com características não usuais. RGO. 2001 out-dez; 49 (4): 210-2.

9. Stafne EC. Diagnóstico Radiográfico Bucal. 4.ed. Rio de Janeiro: Interamericana; 1982.

10. Howe GL. Cirurgia oral menor. 3.ed. São Paulo: Santos; 1990. Amorim RFB, Queiroz SBF, Medeiros AMC, Souza LB.

11. Nóia CF, Oliveira FAC, Pinto JMV, Santos WHM. Odontoma composto. RGO. 2008 abrjun; 56 (2): 213-7.

12. Prata THC, Medice Filho E. Odontomas: neoplasia ou anomalia de desenvolvimento. RPG Rev Pos Grad. 2003 abr-jun; 2 (10): 178-86.

13. Shafer WG, Hine MK, Levy BM. Tratado de Patologia Bucal. 4.ed. Rio de Janeiro: Guanabara Koogan, 1987.

14. Phillipart CALA, Douruv N. Experimental odontomas in osteopetrotic op/ op rats. J Oral Pathol Med. 1994 may; 23 (5): 200-4.

15. Pires LD, Krüger MLB, Viana ES, Kramer PF, Ferreira SL. Odontoma: estado da arte e relato de caso clínico. Stomatos. 2007 jan-jun; 13 (24): 21-9.

16. Lukes SM, Wachter KM. Compound odontoma: a case study. J Dent Hyg. 2003; 77 (1): 47-9.

17. Blinder D, Peleg M, Talcher S. Surgical considerations is cases of large mandibular odontomas located in the mandibular angle. Int $\mathbf{J}$ Oral Maxillofac Surg. 1993 jun; 22 (3): 163-5.

18. Serra-Serra G, Berini-Aytés L, Gay-Escoda C. Erupted odontomas: a report of three cases and review of the literature. Med Oral Patol Cir Bucal. 2009 jun; 14 (6): 299-303.

Submetido em: 16-7-2013

Aceito em: 11-9-2013 